\title{
The first negative Hecke eigenvalue of a Siegel cusp form of genus two
}

\author{
by \\ Winfried Kohnen (Heidelberg) and Jyoti Sengupta (Mumbai)
}

1. Introduction and statement of result. Recently there have been several works on sign changes of Fourier coefficients and Hecke eigenvalues of elliptic cusp forms (cf. e.g. [4, 8, 10, 12, 13]).

Notably in [8] it was shown that if $f$ is a normalized Hecke eigenform of integral weight $k \geq 2$ and level $N \in \mathbb{N}$, and $\lambda(n)(n \in \mathbb{N})$ denote its Hecke eigenvalues, then there exists $n \in \mathbb{N}$ with

$$
n \ll\left(k^{2} N\right)^{29 / 60}
$$

such that $\lambda(n)<0$. Here the constant implied in $\ll$ is absolute and effectively computable. The proof uses convexity estimates for the Hecke $L$-function of $f$ and exploits the Hecke relations satisfied by the $\lambda(n)$.

Let $S_{k}\left(\Gamma_{2}\right)$ be the space of Siegel cusp forms of integral weight $k$ on the group $\Gamma_{2}:=\mathrm{Sp}_{2}(\mathbb{Z}) \subset \mathrm{GL}_{4}(\mathbb{Z})$ and let $F$ be a non-zero eigenfunction of all the Hecke operators $T(n)(n \in \mathbb{N})$ (cf. e.g. [2,7] for details). Denote by $\lambda(n)$ $(n \in \mathbb{N})$ the corresponding eigenvalues.

If $k$ is even and $F$ is contained in the Maass subspace $S_{k}^{*}\left(\Gamma_{2}\right) \subset S_{k}\left(\Gamma_{2}\right)$ (cf. e.g. [5]), it was proved in [3] that $\lambda(n)>0$ for all $n$. On the other hand, if either $k$ is odd, or $k$ is even and $F$ is in the orthogonal complement of $S_{k}^{*}\left(\Gamma_{2}\right)$, then under the validity of the Ramanujan-Petersson conjecture for $F$ (a proof of which was announced in [15]) it was recently shown in [11] that the sequence $(\lambda(n))_{n \in \mathbb{N}}$ indeed changes sign infinitely often.

In the present paper we shall prove

TheOREm. Let $F$ be a non-zero Siegel-Hecke eigenform in $S_{k}\left(\Gamma_{2}\right)$ and suppose that either $k$ is odd, or $k$ is even and $F$ is in the orthogonal complement of $S_{k}^{*}\left(\Gamma_{2}\right)$. Assume that $F$ satisfies the Ramanujan-Petersson conjecture (cf. Sect. 2). Denote by $\lambda(n)(n \in \mathbb{N})$ the eigenvalues of $F$. Then there

2000 Mathematics Subject Classification: Primary 11F46.

Key words and phrases: Siegel modular form, Hecke operator, Maass subspace, Ramanujan-Petersson conjecture. 
exists $n \in \mathbb{N}$ with

$$
n \ll k^{2} \log ^{20} k
$$

such that $\lambda(n)<0$. Here the constant implied in $\ll$ is absolute and effectively computable.

We note that the first case where a form $F$ as above exists is $k=35$ if $k$ is odd and $k=20$ if $k$ is even.

The proof of the Theorem follows a similar pattern to that in [8], with the Hecke $L$-function replaced by the spinor zeta function. However, since the Hecke relations for $\lambda(n)$ are more involved in genus 2 than in the elliptic case, exploiting them naturally turns out to be more difficult.

Notations. If in an estimate we write $\ll$, it is always understood that the implied constant is absolute.

2. Preliminaries on Siegel modular forms. For basic facts on Siegel modular forms we refer to $[2,7,9]$. For $n \in \mathbb{N}$ there is a Hecke operator $T(n)$ on $S_{k}\left(\Gamma_{2}\right)$ given by

$$
F\left|T(n)=\sum_{\gamma \in \Gamma_{2} \backslash \mathcal{O}_{2, n}} F\right|_{k} \gamma
$$

where $\mathcal{O}_{2, n}$ is the set of integral symplectic similitudes of size 4 and scale $n$ and

$$
\left(\left.F\right|_{k} \gamma\right)(Z):=(\operatorname{det} \gamma)^{k / 2} \operatorname{det}(C Z+D)^{-k} F\left((A Z+B)(C Z+D)^{-1}\right)
$$

for

$$
\gamma=\left(\begin{array}{ll}
A & B \\
C & D
\end{array}\right), \quad Z \in \mathcal{H}_{2}=\text { Siegel upper half space of genus } 2 .
$$

Note that our choice of normalization in (2.1) differs from the usual one by the scalar factor $n^{k-3 / 2}$.

The space $S_{k}\left(\Gamma_{2}\right)$ has a basis consisting of common eigenfunctions of all the $T(n)$. The Maass subspace $S_{k}^{*}\left(\Gamma_{2}\right)$ ( $k$ even) is invariant under all Hecke operators.

Let $F$ be a non-zero eigenfunction of all $T(n)$, with $F \mid T(n)=\lambda(n) F$. Then $\lambda(n)$ is real for all $n$.

One has

$$
\sum_{n \geq 1} \lambda(n) n^{-s}=\frac{1}{\zeta(2 s+1)} Z_{F}(s) \quad(\sigma:=\Re(s) \gg 1)
$$

where $Z_{F}(s)$ is the spinor zeta function of $F$, i.e.

$$
Z_{F}(s)=\prod_{p} Z_{F, p}\left(p^{-s}\right)^{-1} \quad(\sigma \gg 1)
$$


with

$$
\begin{aligned}
& Z_{F, p}(X) \\
& \quad:=\left(1-\alpha_{0, p} X\right)\left(1-\alpha_{0, p} \alpha_{1, p} X\right)\left(1-\alpha_{0, p} \alpha_{2, p} X\right)\left(1-\alpha_{0, p} \alpha_{1, p} \alpha_{2, p} X\right)
\end{aligned}
$$

and where $\alpha_{0, p}, \alpha_{1, p}, \alpha_{2, p}$ are "the" Satake $p$-parameters attached to $F$. For details we refer to [1].

Note that due to our normalization one has

$$
\alpha_{0, p}^{2} \alpha_{1, p} \alpha_{2, p}=1 \text {. }
$$

Indeed, in comparison to the "classical" normalization we have replaced the variable $s$ by $s+k-3 / 2$.

The function

$$
Z_{F}^{*}(s):=(2 \pi)^{-s} \Gamma(s+k-3 / 2) \Gamma(s+1 / 2) Z_{F}(s)
$$

has meromorphic continuation to $\mathbb{C}$ and is $(-1)^{k}$-invariant under $s \mapsto 1-s$ (see [1]). It is entire if and only if either $k$ is odd, or $k$ is even and $F$ is in the orthogonal complement of $S_{k}^{*}\left(\Gamma_{2}\right)[6,14]$.

In the latter case the Ramanujan-Petersson conjecture says that

$$
\left|\alpha_{1, p}\right|=\left|\alpha_{2, p}\right|=1 \quad(\forall p)
$$

(a proof was announced in [15]). By (2.5) we then also have

$$
\left|\alpha_{0, p}\right|=1 \quad(\forall p) \text {. }
$$

3. Convexity estimates. Let $F \in S_{k}\left(\Gamma_{2}\right)$ be a non-zero Hecke eigenform with normalized eigenvalues $\lambda(n)(n \in \mathbb{N})$. We assume that either $k$ is odd, or $k$ is even and $F$ is not contained in $S_{k}^{*}\left(\Gamma_{2}\right)$. We also assume (2.6).

The purpose of this section is to derive estimates uniform with respect to $k$ for $Z_{F}(s)$ on lines $s=\delta+i t(t \in \mathbb{R})$ where $0<\delta<1 / 2$. The arguments will be analogous to those given in Sect. 3 of [12] and therefore we will be brief.

Let us write

$$
Z_{F}(s)=\sum_{n \geq 1} a(n) n^{-s} \quad(\sigma \gg 1) .
$$

Then from (2.3), (2.4), (2.6) and (2.7) we obtain

$$
|a(n)| \leq d_{4}(n) \quad(n \geq 1)
$$

where $d_{4}(n)$ is the $n$th coefficient of $\zeta^{4}(s)$. Since $\zeta^{4}(s)$ has a pole at $s=1$ of order 4, a standard Tauberian argument gives

$$
\sum_{x_{0} \leq n \leq x}|a(n)| \ll x \log ^{3} x \quad\left(x_{0}>1\right) .
$$


Using integration by parts for Stieltjes integrals we deduce from (3.1) in a similar way to [12] that

$$
\left|Z_{F}(c+i t)\right| \ll 1+\frac{c}{(c-1)^{4}}
$$

whenever $c>1$.

Next by the functional equation of $Z_{F}^{*}(s)$ we get

$$
\left|Z_{F}(1-s)\right|=(2 \pi)^{2-4 \sigma}\left|\frac{\Gamma(s+k-3 / 2) \Gamma(s+1 / 2)}{\Gamma(-s+k-1 / 2) \Gamma(3 / 2-s)}\right| \cdot\left|Z_{F}(s)\right| .
$$

Putting $s=c+i t$ and observing that $|\Gamma(z)|=|\Gamma(\bar{z})|$, we in particular obtain $\left|Z_{F}(1-c-i t)\right|=(2 \pi)^{2-4 c}\left|\frac{\Gamma(k-3 / 2+c+i t) \Gamma(c+1 / 2+i t)}{\Gamma(k-1 / 2-c+i t) \Gamma(3 / 2-s c+i t)}\right| \cdot\left|Z_{F}(c+i t)\right|$.

We estimate the quotients of $\Gamma$-factors in the same way as in [12] to deduce that

$$
\left|Z_{F}(1-c-i t)\right| \ll|k-1+2 i t|^{2 c-1}|1+i t|^{2 c-1}\left|Z_{F}(c+i t)\right|,
$$

hence

$$
\left|Z_{F}(1-c-i t)\right| \ll k^{2 c-1}|1+i t|^{4 c-2}\left|Z_{F}(c+i t)\right| .
$$

Now put

$$
c:=1+\frac{1}{2 \log k} .
$$

Then from (3.2) we infer that

$$
\left|Z_{F}\left(1+\frac{1}{2 \log k}+i t\right)\right| \ll \log ^{4} k
$$

and therefore combining with (3.3) it follows that

$$
\left|Z_{F}\left(-\frac{1}{2 \log k}+i t\right)\right| \ll k \log ^{4} k \cdot|1+i t|^{2+2 / \log k} .
$$

Let us now recall the following "strong convexity" principle, due to Rademacher (cf. e.g. [12, Sect. 3]).

Lemma 1. Suppose that $g(s)$ is continuous on the closed strip $a \leq \sigma \leq b$ and holomorphic and of finite order on $a<\sigma<b$. Furthermore suppose that

$$
|g(a+i t)| \leq E|P+a+i t|^{\alpha}, \quad|g(b+i t)| \leq F|P+b+i t|^{\beta} .
$$

Here $E$ and $F$ are positive constants and $P, \alpha$ and $\beta$ are real constants that satisfy

$$
P+a>0, \quad \alpha \geq \beta .
$$

Then for all $a<\sigma<b$ we have

$$
|g(s)| \leq\left(E|P+s|^{\alpha}\right)^{(b-\sigma) /(b-a)}\left(F|P+s|^{\beta}\right)^{(\sigma-a) /(b-a)} .
$$


We apply Lemma 1 to $Z_{F}(s)$ with

$$
\begin{gathered}
a=-\frac{1}{2 \log k}, \quad b=P=1+\frac{1}{2 \log k}, \quad E=k \log ^{4} k, \quad F=\log ^{4} k, \\
\alpha=2\left(1+\frac{1}{\log k}\right), \quad \beta=0
\end{gathered}
$$

and $s=\delta+i t$ where $0<\delta<1 / 2$. From (3.4) and (3.5) we then obtain easily

Proposition 1. Let $F \in S_{k}\left(\Gamma_{2}\right)$ be a non-zero Hecke eigenform with normalized eigenvalues $\lambda(n)(n \in \mathbb{N})$. Assume that either $k$ is odd, or $k$ is even and $F \notin S_{k}^{*}\left(\Gamma_{2}\right)$. Let $0<\delta<1 / 2$. Then for all $t \in \mathbb{R}$ one has

$$
\left|Z_{F}(\delta+i t)\right| \ll k^{1-\delta} \log ^{4} k \cdot\left|1+\frac{1}{2 \log k}+\delta+i t\right|^{2+1 / \log k-2 \delta} .
$$

4. An upper bound for sums of eigenvalues. We shall prove

Proposition 2. Let $F \in S_{k}\left(\Gamma_{2}\right)$ be a non-zero Hecke eigenform with normalized eigenvalues $\lambda(n)(n \in \mathbb{N})$. Assume that either $k$ is odd, or $k$ is even and $F \notin S_{k}^{*}\left(\Gamma_{2}\right)$. Also suppose that (2.6) holds. Then

$$
\sum_{n \leq x} \lambda(n) \log ^{2}\left(\frac{x}{n}\right) \ll k \log ^{8} k \cdot x^{2 / 3 \log k} .
$$

Proof. By Perron's formula and (2.2) we have

$$
\sum_{n \leq x} \lambda(n) \log ^{2}\left(\frac{x}{n}\right)=\frac{2}{2 \pi i} \int_{2-i \infty}^{2+i \infty} \frac{1}{\zeta(2 s+1)} Z_{F}(s) \frac{x^{s}}{s^{3}} d s
$$

(cf. [12, Sect. 5]).

Let $\frac{1}{2 \log k}<\delta<1 / 2$. We shift the line of integration to the line $\sigma=\delta$ and recall the well-known estimate (say)

$$
\left|\frac{1}{\zeta(\sigma+i t)}\right| \ll \beta(t)
$$

valid (uniformly) for $\sigma>1$, where

$$
\beta(t):= \begin{cases}1 & \text { if }|t| \leq 10 \\ \log |t| & \text { if }|t|>10\end{cases}
$$

Applying (3.6) we then obtain in a standard way

$$
\begin{aligned}
& \sum_{n \leq x} \lambda(n) \log ^{2}\left(\frac{x}{n}\right) \\
& \quad \ll k^{1-\delta} \log ^{4} k \cdot \int_{-\infty}^{\infty} \beta(t) \frac{\left|1+\frac{1}{2 \log k}+\delta+i t\right|^{2+1 / \log k-2 \delta}}{|\delta+i t|^{3}} d t \cdot x^{\delta} .
\end{aligned}
$$


Note that the integral on the right-hand side of (4.1) is absolutely convergent since $2+1 / \log k-2 \delta<2$ by hypothesis.

We have to estimate this integral from above uniformly in $k$. Replacing $t$ by $-t$, it is sufficient to get an upper bound on

$$
I_{k, \delta}:=\int_{0}^{\infty} \beta(t) \frac{\left|1+\frac{1}{2 \log k}+\delta+i t\right|^{2+1 / \log k-2 \delta}}{|\delta+i t|^{3}} d t .
$$

Note that for $0<B<A$ one has

$$
|A+i t| \leq \frac{A}{B}|B+i t| \quad(\forall t \in \mathbb{R}) .
$$

Applying this with

$$
A:=1+\frac{1}{2 \log k}+\delta, \quad B:=\delta
$$

we see that the integrand in (4.2) is bounded from above by

$$
C_{k, \delta} \beta(t)|\delta+i t|^{-1+1 / \log k-2 \delta}
$$

where

$$
C_{k, \delta}:=\left(1+\frac{1+\frac{1}{2 \log k}}{\delta}\right)^{2+1 / \log k-2 \delta} .
$$

We split up $I_{k, \delta}$ into an integral from 0 to 10 and an integral from 10 to $\infty$. The first integral is clearly bounded by

$$
\ll C_{k, \delta} \delta^{-1+1 / \log k-2 \delta} \text {. }
$$

The second integral is bounded by

$$
\ll C_{k, \delta} \int_{10}^{\infty} \log t \cdot t^{-1+1 / \log k-2 \delta} d t \ll C_{k, \delta}\left(\frac{1}{2 \delta-\frac{1}{\log k}}\right)^{2},
$$

where the last estimate follows by partial integration.

We now choose

$$
\delta:=\frac{2}{3 \log k} .
$$

We then obtain

$$
I_{k, \delta} \ll \log ^{2} k \cdot\left(\log k+\log ^{2} k\right) \ll \log ^{4} k .
$$

Also $k^{1-\delta} \ll k$ (in fact $k^{1-\delta}$ is of the same order of magnitude as $k$ ).

Thus from (4.1) we obtain our assertion.

\section{A lower bound for sums of eigenvalues}

Proposition 3. Let $F \in S_{k}\left(\Gamma_{2}\right)$ be a non-zero Hecke eigenform and assume that either $k$ is odd, or $k$ is even and $F \notin S_{k}^{*}\left(\Gamma_{2}\right)$. Suppose that (2.6) 
holds. Let $\lambda(n)(n \in \mathbb{N})$ be the normalized eigenvalues of $F$ and suppose that $\lambda(n) \geq 0$ for $1 \leq n \leq x$. Then

$$
\sum_{n \leq x} \lambda(n) \log ^{2}\left(\frac{x}{n}\right) \gg \frac{\sqrt{x}}{\log ^{2} x} \quad(x>1) .
$$

Proof. Clearly

$$
\sum_{n \leq x} \lambda(n) \log ^{2}\left(\frac{x}{n}\right) \gg \sum_{n \leq x / 2} \lambda(n),
$$

hence it suffices to show that

$$
\sum_{n \leq x} \lambda(n) \gg \frac{\sqrt{x}}{\log ^{2} x} \quad(x>1) .
$$

By [1], for each prime $p$ the local spinor polynomial $Z_{F, p}(X)$ given by (2.4) is equal to

$$
Z_{F, p}(X)=1-\lambda(p) X+\left(\lambda(p)^{2}-\lambda\left(p^{2}\right)-1 / p\right) X^{2}-\lambda(p) X^{3}+X^{4},
$$

hence by $(2.2)$ we have

$$
\frac{1-\frac{1}{p} X^{2}}{Z_{F, p}(X)}=\sum_{n \geq 0} \lambda\left(p^{n}\right) X^{n} .
$$

Clearly (5.2) is equivalent to saying that

$$
\begin{aligned}
\lambda\left(p^{n}\right)= & \lambda(p) \lambda\left(p^{n-1}\right)-\left(\lambda(p)^{2}-\lambda\left(p^{2}\right)-1 / p\right) \lambda\left(p^{n-2}\right) \\
& +\lambda(p) \lambda\left(p^{n-3}\right)-\lambda\left(p^{n-4}\right)
\end{aligned}
$$

for all $n \geq 0$, with the convention that $\lambda\left(p^{n}\right)=0$ for $n<0$.

Note that (2.4), (2.6), (2.7) and (5.2) imply that

$$
|\lambda(p)|,\left|\lambda\left(p^{2}\right)\right|,\left|\lambda\left(p^{3}\right)\right| \ll 1 .
$$

To prove (5.1), bearing in mind that $\lambda(n) \geq 0$ for $n \leq x$, let us write

$$
\sum_{n \leq x} \lambda(n) \geq \sum_{p, q \leq \sqrt[4]{x}} \lambda\left(p^{2} q^{2}\right)+\sum_{p, q \leq \sqrt[4]{x}} \lambda\left(p^{2} q\right)+\sum_{p, q \leq \sqrt[4]{x}} \lambda(p q)
$$

where on the right-hand side $p$ and $q$ run over primes.

Taking $n=4$ in (5.3) we obtain

$$
\lambda\left(p^{4}\right)=\lambda\left(p^{2}\right)^{2}+\lambda(p) \lambda\left(p^{3}\right)+\lambda\left(p^{2}\right)\left(-\lambda(p)^{2}+1 / p\right)+\lambda(p)^{2}-1 .
$$

Similarly, for $n=3$ we find that

$$
\lambda\left(p^{3}\right)=\lambda(p)\left(2 \lambda\left(p^{2}\right)+1+1 / p-\lambda(p)^{2}\right) .
$$

From (5.6), observing (5.4) we see that

$$
\lambda\left(p^{4}\right) \gg \lambda\left(p^{2}\right)^{2}-c_{1}
$$


where $c_{1}>0$ is an absolute constant. Thus

$$
\sum_{p, q \leq \sqrt[4]{x}} \lambda\left(p^{2} q^{2}\right) \gg\left(\sum_{p \leq \sqrt[4]{x}} \lambda\left(p^{2}\right)\right)^{2}-c_{1} \pi(\sqrt[4]{x})
$$

where as usual $\pi(x)(x>1)$ denotes the number of primes $p \leq x$.

Next, from (5.7) taking into account (5.4) we see that

$$
\lambda\left(p^{3}\right) \gg \lambda(p) \lambda\left(p^{2}\right)-c_{2}
$$

where $c_{2}>0$ is an absolute constant. Hence

$$
\sum_{p, q \leq \sqrt[4]{x}} \lambda\left(p^{2} q\right) \gg\left(\sum_{p \leq \sqrt[4]{x}} \lambda\left(p^{2}\right)\right)\left(\sum_{p \leq \sqrt[4]{x}} \lambda(p)\right)-c_{2} \pi(\sqrt[4]{x}) .
$$

We finally look at the sum

$$
\sum_{p, q \leq \sqrt[4]{x}} \lambda(p q)
$$

in (5.5). For $p \leq \sqrt[4]{x}$ the quantities $\lambda\left(p^{3}\right), \lambda\left(p^{2}\right)$ and $\lambda(p)$ are non-negative, hence we deduce from (5.7) for such $p$ that

$$
\lambda\left(p^{2}\right) \gg \lambda(p)^{2}-c_{3}
$$

where $c_{3}>0$ is an absolute constant. Therefore as before

$$
\sum_{p, q \leq \sqrt[4]{x}} \lambda(p q) \gg\left(\sum_{p \leq \sqrt[4]{x}} \lambda(p)\right)^{2}-c_{3} \pi(\sqrt[4]{x}) .
$$

Combining (5.8), (5.9) and (5.10) we infer from (5.5) that

$$
\sum_{n \leq x} \lambda(n) \gg\left(\sum_{p \leq \sqrt[4]{x}} \lambda\left(p^{2}\right)+\sum_{p \leq \sqrt[4]{x}} \lambda(p)\right)^{2}-c \pi(\sqrt[4]{x})
$$

where $c>0$ is an absolute constant.

We now claim that $\lambda\left(p^{2}\right)$ and $\lambda(p)$ cannot be simultaneously small for $p \leq \sqrt[4]{x}$. Indeed, otherwise $\lambda\left(p^{3}\right)$ would also be small, by (5.7), and then (5.6) would give a contradiction since $\lambda\left(p^{4}\right) \geq 0$ by hypothesis. Thus there exists an absolute constant $\alpha>0$ such that

$$
\lambda\left(p^{2}\right)+\lambda(p) \geq \alpha \quad(p \leq \sqrt[4]{x}) .
$$

From (5.11) we now conclude using the prime number theorem that

$$
\sum_{n \leq x} \lambda(n) \gg \frac{\sqrt{x}}{\log ^{2} x}
$$

as claimed. 
6. Proof of Theorem. Assuming that $\lambda(n) \geq 0$ for $n \leq x$, we infer from Propositions 2 and 3 that

$$
\frac{\sqrt{x}}{\log ^{2} x} \ll k \log ^{8} k \cdot x^{2 / 3 \log k} \quad(x>1) .
$$

Clearly for $x$ large this is a contradiction.

To get an explicit bound, quoting the more general Lemma 4 in [4] we see that (6.1) implies that

$$
x \ll\left(\frac{A}{\delta^{2}}\right)^{1 / \delta} \log ^{2 / \delta}\left(\frac{A}{\delta^{2}}\right)
$$

where

$$
A:=k \log ^{8} k, \quad \delta:=\frac{1}{2}-\frac{2}{3 \log k} .
$$

We have

$$
\frac{1}{\delta}=2+\frac{8}{3 \log k-4} .
$$

Hence

$$
A^{1 / \delta}=\left(k \log ^{8} k\right)^{2+8 /(3 \log k-4)} \ll k^{2} \log ^{16} k
$$

and

$$
\log ^{2 / \delta}\left(\frac{A}{\delta^{2}}\right) \ll \log ^{2 / \delta} A \ll \log ^{4} k .
$$

Thus (6.2) implies that

$$
x \ll k^{2} \log ^{20} k .
$$

Therefore we obtain the assertion of the Theorem.

\section{References}

[1] A. N. Andrianov, Euler products corresponding to Siegel modular forms of genus 2, Russian Math. Surveys 29 (1974), 45-116.

[2] -, Quadratic Forms and Hecke Operators, Grundlehren Math. Wiss. 286, Springer, Berlin, 1987.

[3] S. Breulmann, On Hecke eigenforms in the Maass space, Math. Z. 232 (1999), 527530.

[4] Y. J. Choie and W. Kohnen, The first sign change of Fourier coefficients of cusp forms, preprint, 2006.

[5] M. Eichler and D. Zagier, The Theory of Jacobi Forms, Progr. Math. 55, Birkhäuser, Boston, 1985.

[6] S. A. Evdokimov, A characterization of the Maass space of Siegel cusp forms of genus 2, Mat. Sb. 112 (1980), 133-142 (in Russian).

[7] E. Freitag, Siegelsche Modulformen, Grundlehren Math. Wiss. 254, Springer, Berlin, 1983.

[8] H. Iwaniec, W. Kohnen and J. Sengupta, The first negative Hecke eigenvalue, Int. J. Number Theory, to appear. 
[9] H. Klingen, Introductory Lectures on Siegel Modular Forms, Cambridge Stud. Adv. Math. 20, Cambridge Univ. Press, 1999.

[10] M. Knopp, W. Kohnen and W. Pribitkin, On the signs of Fourier coefficients of cusp forms, Ramanujan J. 7 (2003), 269-277.

[11] W. Kohnen, Sign changes of Hecke eigenvalues of Siegel cusp forms of genus two, Proc. Amer. Math. Soc. 135 (2007), 997-999.

[12] W. Kohnen, Y.-K. Lau and J. Sengupta, On the first sign change of Hecke eigenvalues of newforms, Math. Z. 254 (2006), 173-184.

[13] W. Kohnen and I. Shparlinski, On the number of sign changes of Hecke eigenvalues of newforms, preprint, 2006.

[14] T. Oda, On the poles of Andrianov L-functions, Math. Ann. 256 (1981), 323-340.

[15] R. Weissauer, The Ramanujan conjecture for genus 2 Siegel modular forms (an application of the trace formula), preprint, Mannheim, 1993.

Mathematisches Institut, INF 288

Universität Heidelberg

D-69120 Heidelberg, Germany

E-mail: winfried@mathi.uni-heidelberg.de
School of Mathematics

T.I.F.R.

Homi Bhabha Road

400005 Mumbai, India

E-mail: sengupta@math.tifr.res.in

Received on 2.11.2006

and in revised form on 10.4.2007 